\title{
Transcription Initiation Factor TFIID Subunit 8
}

National Cancer Institute

\section{Source}

National Cancer Institute. Transcription Initiation Factor T FIID Subunit 8. NCI Thesaurus.

Code C30070.

Transcription initiation factor TFIID subunit 8 (310 aa, $\sim 34 \mathrm{kDa}$ ) is encoded by the human

TAF8 gene. This protein is involved in both transcriptional initiation and cellular

differentiation. 